Forma mentis networks quantify crucial differences in STEM perception between students and experts

\title{
Massimo Stella*
}

Institute for Complex Systems Simulation, University of Southampton, UK and Complex Science Consulting, Italy

Sarah De Nigris

Institute for Web Science and Technologies, University of Koblenz-Landau, Germany

Aleksandra Aloric

Scientific Computing Laboratory, Center for the Study of Complex Systems, Institute of Physics Belgrade, Serbia

Cynthia S. Q. Siew

Department of Psychology, University of Warwick, UK and Department of Psychology, National University of Singapore, Singapore

Author Note

*Corresponding author: Massimo Stella, massimo.stella@inbox.com 


\begin{abstract}
In order to investigate the stances of high school students and researchers toward STEM subjects, we introduce the methodology of forma mentis networks - free association networks enriched with affective attributes that represent how people conceptually perceive and structure their stance toward a given topic. In this paper, we constructed separate forma mentis networks for $\left(N_{s}=159\right)$ Italian high school students and $\left(N_{r}=59\right)$ interdisciplinary professionals and researchers in order to investigate how these groups differed in their mental associations and emotional perceptions of STEM subjects. At the global scale, STEM concepts occupied central positions in students' forma mentis network, suggesting that students understood the general importance of such topics in science. At a microscopic scale, although the concept of "science" was positively perceived in both the students' and professionals' forma mentis networks, students not only perceived STEM concepts such as "physics" and "mathematics" as negative but also associated them with other negative STEM-related concepts. This aura of negative emotional associations towards quantitative STEM subjects was absent in professionals. Cross-validation with external datasets suggested that the negative emotional aura in the forma mentis network of students might be attributed to science anxiety. Further consideration of the semantic associates of maths and physics indicated that their negative aura may originate from a negative, dry perception of the technical methodology and quantitative tools frequently taught in these subjects (e.g., "function", "integral"). Whereas students associated mathematics and physics with quantitative tools, professionals linked the same disciplines to more general and creative aspects of science and displayed a positive stance towards these concepts. Overall, our results underline the crucial importance of emphasising nontechnical and applied aspects in the teaching of quantitative disciplines, highlighting the necessity of establishing interdisciplinary links between science, the complexity of the real-world and creativity in order to enhance the impact of STEM education and outreach activities.
\end{abstract}

Keywords: stance detection, complex networks, STEM, education, psychology, psycholinguistics 
Forma mentis networks quantify crucial differences in STEM perception between students and experts

\section{Introduction}

Increasing evidence indicates that many students develop a negative perception of STEM subjects before ending high school (Krapp \& Prenzel, 2011; Osborne, Simon, \& Collins, 2003; Valenti, Masnick, Cox, \& Osman, 2016). Mathematics is viewed as a difficult subject, physics is perceived as too abstract, and statistics is often considered an uninterpretable black box (Ashcraft, 2002; Valenti et al., 2016). A growing disinterest of students towards Science, Technology, Engineering and Mathematics (STEM) disciplines represents an unseen societal cost, as it translates into a lower interest in pursuing technological and scientific careers which are increasingly found to positively correlate with job growth, higher employment rates, societal innovation through functional literacy and economic development (Marginson, Tytler, Freeman, \& Roberts, 2013; Rothwell, 2013). Before addressing students' (mis)perception of STEM subjects, educators and policymakers first need to understand the detailed nature of the students' opinions and beliefs about science.

With this aim, this paper capitalizes on an innovative combination of methods from network science and cognitive science to examine the perception of STEM subjects among a population of students and another population of researchers. Specifically, we introduce the methodology of forma mentis networks (FMNs), which represent the associative structure of concepts as well as their valence, and show how FMNs can be harnessed to study a population's stance toward a given topic.

Forma mentis networks are constructed from language data. Linguistic information, such as text or speech, often conveys the opinion or attitude of an individual towards a given entity (Aitchison, 2012; Siegel, 2015), e.g., a human reading a blog can understand which posts are in favor or against a given political view. However, stance detection, i.e., detecting the stance of an individual or population from language (Gray \& Biber, 2012; S. Mohammad, Kiritchenko, Sobhani, Zhu, \& Cherry, 2016), is not an easy task. 
In the past few decades, stance detection has spurred research at the interface of psycholinguistics and computer science, which has led to the development of a variety of methodologies through the human coding of grammatical features of text (Biber \& Finegan, 1989; Gray \& Biber, 2012), e.g. the use of specific adverbs or writing styles. Such approaches are centralised, in that they require a human coder, e.g. a linguist, to parse the input and detect the features that are important for the identification of the author's stance.

Centralised human coding cannot deal with the large volumes of linguistic data that are increasingly available, for instance from social media platforms (Stella, Ferrara, \& De Domenico, 2018). This motivated the development of automatic techniques for detecting stance based on computer science approaches such as machine learning (S. M. Mohammad, Sobhani, \& Kiritchenko, 2017; S. Mohammad et al., 2016; Somasundaran \& Wiebe, 2010). A notable example is a recent approach by Mohammad and colleagues (S. M. Mohammad et al., 2017), who deployed machine learning of sentiment features and word embeddings for successfully detecting the stance of individual messages from social media. The results of Mohammad and colleagues clearly show that stance detection is not the same as sentiment analysis. Sentiment analysis determines the specific affect valence of a given piece of linguistic data, i.e. how universally positive/negative/neutral are the concepts elicited by a given portion of text. Instead, stance emerges at a higher level as a non-trivial combination of different patterns of affect and sentiment. For example, the sentence "The dictator who killed my relatives has been finally executed" includes concepts of negative sentiment (e.g. dictator, executions, etc.), but nonetheless elicits a positive stance towards the execution itself. It is important to underline the additional complexity of stance in comparison with sentiment, as affective patterns in the language need to be integrated with additional contextual information before achieving an accurate classification of stance itself (S. M. Mohammad et al., 2017).

Although machine learning approaches are powerful in underlining the different psychological dimensions of stance in terms of context and sentiment (S. M. Mohammad 
et al., 2017), these automatic techniques have at least two limitations: (i) performance depends on the availability and quality of large-scale annotated training data, and (ii) machine learning builds "black-box" representations of data that cannot be directly accessed or interpreted. Due to these two elements, supervised learning approaches to stance detection are not yet widespread in the cognitive sciences, although they represent an interesting and powerful perspective for future work.

Beyond supervised learning, network models stand as a promising avenue to the investigation of cognitive and linguistic data, leading to the emergence of the field of cognitive network science (Siew, Wulff, Beckage, \& Kenett, 2018). Network models of language are often interpreted as descriptive representations of the mental lexicon, a repository of linguistic and semantic knowledge in human memory (Aitchison, 2012). Decades of research in psycholinguistics has shown that the mental lexicon is not a static list of words, e.g. a dictionary, but it rather is a dynamical system optimized for cognitive computing which stores and processes individual concepts together with their associated linguistic data, e.g. semantic overlap in meaning (Steyvers \& Tenenbaum, 2005), phonological similarities (Vitevitch, 2008; Vitevitch, Siew, \& Castro, 2018), syntactic relationships between word categories (Stella, Beckage, Brede, \&

De Domenico, 2018). Psycholinguistic evidence has shown that the associative structure of the mental lexicon influences language processes such as word learning (Hills \& Siew, 2018; Stella, 2019; Stella, Beckage, \& Brede, 2017) and processing (De Deyne, Navarro, \& Storms, 2013; De Deyne, Navarro, Perfors, Brysbaert, \& Storms, 2018; Kenett, Levi, Anaki, \& Faust, 2017; Steyvers \& Tenenbaum, 2005). This strong link between mental lexicon structure and language usage promoted the use of network models for a variety of processes such as the discovery of writing styles from word co-occurrences in texts (Amancio, 2015) or predicting the creativity of individuals (Kenett, Anaki, \& Faust, 2014; Kenett et al., 2018), their curiosity (Zurn \& Bassett, 2018), their openness to new experience (Christensen, Kenett, Cotter, Beaty, \& Silvia, 2018), their expertise in a given domain (Siew, 2018; Valenzuela Castellanos, Pérez Villalobos, Bustos, \& Salcedo Lagos, 2018) and their perceived anxiety toward a topic (Siew, McCartney, \& 
Vitevitch, 2019). Forma mentis networks rely on the framework of cognitive network science to represent the associative and emotional structure of concepts in the mental lexicon.

One of the main ingredients of FMNs is free association data to specify the connections between concepts. Indeed, free associations represent a powerful and meaningful way of building network models of the mental lexicon (De Deyne et al., 2013; De Deyne et al., 2018; Kenett et al., 2017; Kenett et al., 2018). Free associations are obtained empirically from experiments where participants have to produce associates when primed with a cue word. Hence, free associations are largely free from any specific semantic definition (e.g., synonyms). Previous work (Stella et al., 2017; Stella, Beckage, Brede, \& De Domenico, 2018) has shown that free associations partially overlap with other semantic word-word similarities such as synonyms (i.e., two words sharing the same meaning in a given context) or generalisations (i.e., a concept being a special type of another word) but also display a small overlap with phonological similarities among words (e.g., when pronunciations differ in one phoneme).

Forma mentis networks combine free associations with affective patterns of concepts. In a FMN, nodes represent concepts or words, links indicate free associations provided by a given population and every node has a valence attribute (Adelman \& Estes, 2013; Posner, Russell, \& Peterson, 2005; Warriner, Kuperman, \& Brysbaert, 2013) that represent how the population perceives a given concept or word (positive, negative, or neutral). Recent psycholinguistic evidence has shown that the emotional valence of words influences language processing and memory (Adelman \& Estes, 2013; Gaillard et al., 2006), highlighting an important link between affect and the cognitive mechanisms of language processing in the mental lexicon. Therefore, representing knowledge and sentiment combined in a forma mentis network gives access to the structure of the aggregated mental lexicon and affect of a given population.

We emphasize that the addition of valence attributes and the adoption of free associations makes forma mentis networks different from conceptual maps (Ismo T. Koponen \& Nousiainen, 2018, 2019; Ismo T Koponen \& Pehkonen, 2010; 
Sayama, Cramer, Porter, Sheetz, \& Uzzo, 2016), which represent important network models of knowledge acquisition and structuring during learning but do not incorporate information about how learners perceive individual conceptual units. Another difference is that conceptual maps are often based on concept co-occurrence in a syllabus and therefore capture temporal information (Ismo T. Koponen \& Nousiainen, 2019), which is not present in a forma mentis network.

In this paper, we investigated the attitude of students and researchers towards science and STEM subjects through this innovative combination of tools from psycholinguistics and network science to quantify their stances toward STEM subjects. Through the comparison of the FMN of students and research professionals, we provide quantitative evidence for sharp differences in the perception of STEM among the two different groups. Specifically, the combination of conceptual associations and affect patterns allowed us to identify and paint a richer picture of the disaffection towards mathematics and physics exhibited by students and absent in STEM professionals.

\section{Methods}

\section{Participants}

We collected data from 159 students and 59 researchers. Students were selected from three different Italian high schools, without consideration of their grades in STEM subjects. All students were in their final year of high school, with ages ranging between 18 and 19 years (mode: 18 years). In order to build a sample representative of the national Italian student population in high schools, entire classes were selected for testing, to ensure a mixture of socio-economic backgrounds and STEM proficiency levels. The distribution of genders was roughly evenly distributed between female (53\%) and male (47\%) students.

Researchers were early career scientists, which included doctoral students and post-doctoral researchers from diverse backgrounds and who apply quantitative tools originating in the fields of mathematics, physics and computer science to study emerging phenomena in complex systems ranging from biological to socioeconomic 
systems. Hence, all the interviewed researchers possessed advanced training and expertise in STEM and were actively pursuing a professional career in science. The age of the interviewed researchers ranged between 24 and 39 years, with a mode of 29 years. The distribution of genders was roughly evenly distributed between male (56\%) and female (44\%) researchers.

\section{Cognitive tasks}

Each participant took part in a survey composed of two tasks: (i) a free association task and (ii) a valence evaluation task. Participants were given precise instructions about the study before proceeding. Participants were then asked to provide informed consent if they agreed to take part in the study by signing a consent form that described essential points about privacy and ethics in accordance with the Italian Association of Psychology (https://www.aipass.org/node/11560 - Last accessed: 18 February 2019). All consent forms were gathered at the end of the study and are available upon inquiry to the first author.

In the free association task, each participant was presented with a list of 50 cue words. In order to investigate attitudes toward key STEM subjects, 10 out of the 50 cue words were present in all participants' lists. These words were: mathematics, complex, physics, chemistry, system, biology, life, art, school and university. In Italian, these words were translated as: matematica, complesso, fisica, chimica, sistema, biologia, vita, arte, scuola and università. Although "art" is not a STEM subject, its inclusion in the list of essential words was meant to provide some comparison between the humanities and technical subjects. Additionally, adding art to the list of essential words provides a way to probe students' perception of connections between STEM subjects and creativity, a link that has been investigated in previous studies about attitudes towards STEM (Valenti et al., 2016). The other 40 cue words were drawn at random from a subsample of STEM-focused 390 words. The pool of 390 potential cue words was obtained by considering the highest frequency non-stop words from the Wikipedia webpages about "Complex System", "Physics", "Mathematics", "Biology", "Chemistry" 
and "Psychology" (as accessed on: 15 January 2017).

Participants were randomly assigned to one of the 50 files containing a different random realisation with 50 of the previously described words. The order of words in each list was scrambled with the aim of reducing recency effects or other associative biases due to the order of cues.

In order to obtain denser networks of free associations, we used the continuous free association task, which has been shown to provide higher quality data that could account for more variance in lexical retrieval tasks (De Deyne et al., 2013). In the continuous free association task, each participant generated three associative responses to each item in the list of 50 cue words. The association task took place in a lab setting, with each participant filling in electronic forms on a computer terminal while under supervision. Forms that contained more than 25 percent blank responses were discarded. This occurred in roughly $2 \%$ of the cases. The first three associates in each form were discarded in order to minimize potential priming effects that might follow the given instructions. The association task lasted for 10 minutes, followed by a short break.

In the second task, we collected the valence of each cue and for all associations from the free association task. Participants were asked to rate the valence of each cue and their associated responses using a Likert scale ranging from 1 (very negative) to 5 (very positive), with neutrality being represented either by a blank space or by a score

of 3. Participants completed this task in about 10 minutes. Those participants who did not finish the task in 10 minutes left the remaining spaces blank. Forms that contained more than 25 percent blank responses were discarded. This occurred in roughly $3 \%$ of the cases. Data collection was conducted anonymously, such that no demographic or educational data was obtained from the participants and directly linked to the filled forms.

\section{Data cleaning and network building}

Associative responses were converted to lowercase letters and checked automatically and manually for common spelling mistakes. The automatic spell 
checkers used were based on Google Translate and Wolfram's Mathematica 11.3 (manufactured by Wolfram Research, Champaign, US). Different word forms were manually converted to match their singular forms (e.g. in English "muscles" was changed to "muscle") and composite responses were changed to single-word forms (e.g. in Italian "da dove" was changed to "dove").

A forma mentis network was constructed such that nodes represented lexical items and edges indicated free associations between words. Two networks of free associations were constructed from the students' data, one network where only associations provided by at least two different participants were considered $\mathcal{N}_{S}^{C}$ (filtered) and a second network where no filtering was performed $\mathcal{N}_{S}$. Given the considerably smaller sample size of researchers, only a single unfiltered network of free associations was constructed $\mathcal{N}_{R}$. We note that considering idiosyncratic associations, i.e., associations provided by a single participant, like in $\mathcal{N}_{S}$, is common practice when working with free association data obtained from small samples (C. B. Cramer, Porter, Sayama, Sheetz, \& Uzzo, 2018; Ismo T. Koponen \& Nousiainen, 2018; Ismo T Koponen \& Pehkonen, 2010) and they are still considered to be insightful of cognitive patterns (Nelson, McEvoy, \& Schreiber, 2004) (see also the Appendix). Each node in the network was also assigned a valence score and an attribute ("positive", "neutral", or "negative").

In the remainder of the paper, forma mentis network refers to the network representation that simultaneously represents conceptual knowledge derived from free associations and the emotional perceptions of those concepts among a given population (i.e., students or researchers).

\section{Statistical analysis of word valence}

In order to categorize positive, neutral and negative concepts we used a non-parametric statistical test (Kruskall-Wallis test). The statistical test was used to assess whether the scores attributed to word $i$, namely $w_{i}$, had a lower, compatible, or higher median valence as compared to the remaining distribution of valence scores, in formulas $\bigcup_{j \neq i} w_{j}$. Non-parametric testing was used because the original distribution of 
valence scores $\bigcup_{j} w_{j}$ were skewed with a heavy left tail (Pearson's skewness coefficient $s_{s}=3\left(\right.$ mean $_{s}-$ median $\left._{s}\right) / \sigma=1.39$ for students' data and $s_{r}=1.45$ for researchers $)$. Concepts which had a median valence score lower than the rest, according to a Kruskall-Wallis test with significance level $\alpha=0.1$, were labelled as negative. Concepts which had a median valence score higher than the rest, according to a Kruskall-Wallis test with significance level $\alpha=0.1$, were labelled as positive. Remaining concepts were labelled as neutral.

\section{Defining valence beyond lexical items: Valence "auras"}

Valence is a commonly used feature in psycholinguistic models that assesses the sentiment of a given text (Posner et al., 2005; Siegel, 2015). At the word level, the valence of a word represents the positive, neutral, or negative connotation is elicited by the word in a given population. Hence, valence is a feature of individual words, and does not further consider the way in which words are associated with each other.

Combining free associations with their valence in forma mentis networks naturally provides a way of extending the concept of word valence to a given cluster of associated words. We introduce the concept of valence aura, which identifies the valence of the immediate neighbors of a word on a network of free associations. A concept has a negative valence aura if the given concept is associated with more negative concepts than it is associated with positive concepts. On the other hand, a concept has a positive valence aura if the given concept is associated with more positive concepts than it is associated with negative concepts. The polarity of an aura is determined by the most frequent valence of words in the neighborhood (following a majority rule). It is important to note that positively valenced words could have either a negative or positive valence aura, and negatively valenced words could have either a negative or positive valence aura. Hence, valence auras provide us with a way of juxtaposing or contrasting sentiment polarities with consideration of the structural organisation of knowledge beyond how individual concepts are valenced in isolation.

We use the methodology of valence auras to investigate potential differences in the 
way that students and researchers structure their conceptual knowledge and the role of the perceived valence of those concepts. Firstly, within a given population, it would be interesting to assess the tendency of positive concepts to be associated with other positive concepts (i.e., to have a positive valence aura), and equivalently for negative concepts (i.e. negative concepts with other negative ones). This would bolster the idea that sentiment polarities of individual words have the potential of influencing the structural organisation of semantic memory. Secondly, and more importantly, there might be different tendencies to surround positive concepts with positive or negative auras between students and researchers. Differences in the mixing of (individual) word valence and word auras between the forma mentis networks of different populations could potentially highlight important differences in the organisation and perception of knowledge between such populations.

\section{Validation of the definition of auras through additional psycholinguistic data}

In order to validate our operationalization of valence auras and the valence ratings collected in the present study, we used external datasets of word valence for comparison. For English words in the researchers' forma mentis network, we used the affective ratings by Warriner and colleagues (Warriner et al., 2013), whereas for Italian words in the students' forma mentis network we used the valence norms recently gathered by Fairfield and colleagues (Fairfield, Ambrosini, Mammarella, \& Montefinese, 2017). Kendall tau correlation tests indicate that in both cases there is a statistically significant positive correlation (at $\alpha=0.1$ ) between the mean valence scores collected in the current study and the ones obtained from previous investigations (for students: $\tau=0.51, p<10^{-5}$; for researchers: $\left.\tau=0.38, p<10^{-5}\right)$. For students, the dataset by Fairfield et al. contained valence scores for only 491 of the 4483 words in the Italian unfiltered forma mentis network. For researchers, the overlap between our dataset and Warriner and colleagues' was higher, covering 1173 of the 1616 words in the English unfiltered forma mentis network.

In the following, we will not use directly the mean valence scores gathered from 
students or researchers but rather valence attributes (i.e., positive, neutral, or negative) to define the valence auras of words. In the results section, we will show that our retrieved valence attributes are compatible with the valence scores obtained by other studies.

\section{Results}

Network structure and valence identify auras, which in turn identify words of extreme valence and arousal

The operationalization of valence auras as reported in the Methods section combines network structure with the valence of individual words. Does this combination of topological and valence information provide further insights into students and researchers' perception of STEM subjects? In order to answer this question, we compared the mean valence and arousal scores from external psycholinguistic datasets (cf. Methods) of negative words with either positive or negative auras, and positive words with either positive or negative auras. Recall that auras were defined by using the valence ratings and network structures obtained from the cognitive tasks described above, whereas mean valence scores come from external sources (i.e., the Fairfield (Fairfield et al., 2017) and Warriner (Warriner et al., 2013) databases).

Figure 1 reports the mean valence and arousal of words from the students' forma mentis network. In the students' FMN, negative words surrounded by a negative aura have a lower mean valence (based on an external dataset, cf. Methods) as compared to negative words surrounded by any aura. This difference was statistically significant at the 0.1 significance level $\alpha$ (Kruskal-Wallis, $N=116, s=2.8994, p=0.088$ ). The difference in mean valence between positive words surrounded by any aura and positive words surrounded by a positive aura was not statistically significant at the $\alpha=0.1$ level (Kruskal-Wallis, $N=238, s=2.2891, p=0.1314$ ). The forma mentis network of students also highlighted an interesting difference in the mean arousal of negative words surrounded by a negative aura as compared to negative words surrounded by any aura (cf. Figure 1, left plot). Negative words surrounded by a negative aura elicited a 
stronger arousal as compared to negative words surrounded by any aura, and the difference was statistically significant at the $\alpha=0.1$ level (Kruskal-Wallis, $N=116$, $s=2.7338, p=0.0984)$. A threshold of 0.1 was chosen in order to cope with the limited overlap of our data with the external databases.

The above differences suggest that negative auras correspond to a boosted arousal when surrounding words of negative valence. Positive words did not elicit any analogous difference. No statistically significant difference was found among words in the researchers' forma mentis network.

These results indicate that in the organisation of STEM-related concepts as represented by the forma mentis network, negative concepts surrounded by a negative aura are in general perceived as more negative and elicit higher arousal than concepts surrounded by any aura. Since the arousal scores might be a by-product of valence in rating experiments (Adelman \& Estes, 2013), negative words surrounded by a negative aura represent negative concepts that can activate or be activated by other negative concepts and thus lead to an increase in arousal and emotional intensity. The above analysis presents quantitative evidence showing that our operationalization of valence auras of an individual word's direct neighbors in an associative network can highlight additional affective patterns that valence scores of individual words cannot.

The unfiltered forma mentis network of researchers is smaller, less connected and has fewer negative words than the students' forma mentis network. Hence, no significant differences were found at the $\alpha=0.1$ level of significance (Kruskal Wallis, for positive words: $s=1.0674, p=0.3033$; for negative words: $s=0.0574, p=0.8119)$. We hypothesized that this is due to the forma mentis network of researchers lacking the resolution or power to detect distinct affective patterns.

\section{Forma mentis networks show assortative mixing of word valence}

As described above, in a forma mentis network, each word has a valence attribute (e.g. "positive" or "negative"). Links represent associations between two concepts but also between their respective valence attributes (e.g., there can be a link connecting a 

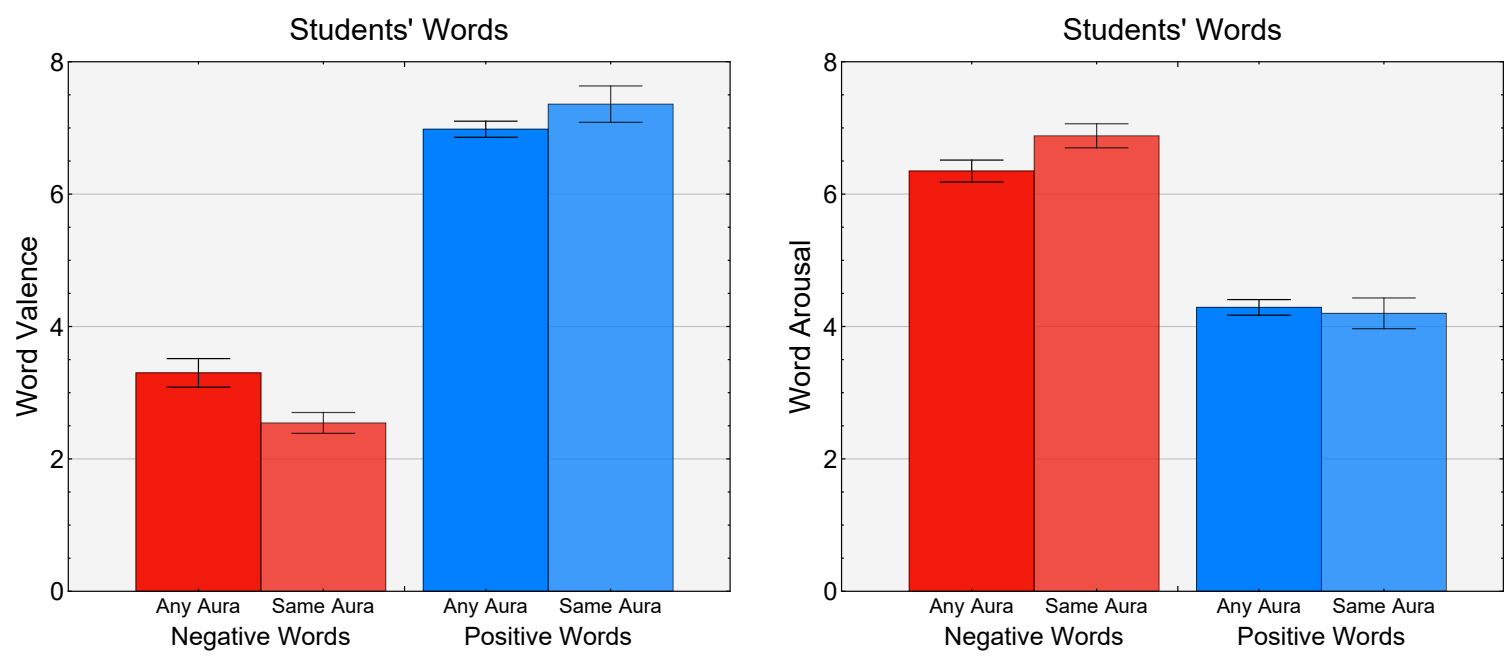

Figure 1. Valence auras identify more extreme negative words in the students' population. Mean valence (left plot) from external psycholinguistic datasets of negative/positive words either surrounded by any valence auras (full colours) or by the same aura (lighter colours). In the students' forma mentis network, negative words surrounded by negative auras are perceived as more strongly negative on average as compared to negative words surrounded by any aura. Negative words surrounded by a negative aura had higher arousal than negative words with any aura (right plot). No difference was found for positive words.

"positive" concept to a "negative" concept). Investigating the assortative or disassortative mixing of valence attributes across network links can shed light on potential trends in students' and researchers' structural and emotional organisation of knowledge.

In the unfiltered network of student associations $\mathcal{N}_{S}$, a Kendall Tau test between the valence attributes of links' endpoints reveals a statistically significant positive correlation $\tau=0.163, p<10^{-5}$. This value might indicate that students tend to associate positive (negative) concepts to other positive (negative) concepts. However, comparison with a reference null model is necessary in order to assess the relative strength of the above correlation and test whether it might be a direct consequence of either the distribution of node degree or the counts of positive, neutral, or negative attributes. We used as null reference a configuration model fixing both the empirical 
degree distribution and the valence attributes of words in the original network but randomising links. An average Kendall Tau of $\tau_{r}=-0.0001(p>0.310)$ was obtained over 50 independent realisations of the null model. As the empirical correlation $\tau=0.163$ was several orders of magnitude larger than random expectation, this indicated that there was a strong tendency for students to associate positive (negative) concepts with other positive (negative) concepts independently of the distributions of either degree or valence attributes. We found a similar pattern in the way that researchers organised and perceived their STEM knowledge (empirical Kendal Tau $\tau=0.116, p<10^{-5}$, reference null model $\tau_{r}=0.027, p>0.112$ ).

\section{Forma mentis networks indicate clustering of word valence and valence auras}

In this section we investigated whether there was a tendency for words to be surrounded by other words with the same valence. To do this, we attributed arbitrary scores to valence attributes, i.e. $-m$ to negative words, 0 to neutral words and $+m$ to positive words. We used $m=1$ for convenience, although our correlation analysis does not depend on the specific value of $m$.

In the students' forma mentis network $\mathcal{N}_{S}$, a Kendall Tau test between the valence attributes of a word and the average valence attributes of its neighbors revealed a statistically significant positive correlation $\tau=0.385, p<10^{-5}$. With 50 independent realisations of a configuration null model with fixed word attributes and degrees but random associations, we found an average correlation of $\tau_{r}=0.053(p=0.060)$. A similar result was found for the researchers' forma mentis network $\mathcal{N}_{R}$, where the empirical correlation value $\left(\tau=0.323, p<10^{-5}\right)$ was considerably higher than random expectation $\left(\tau_{r}=0.060, p=0.056\right)$.

Given that the empirical correlations were several orders of magnitude larger than the reference values, our results showed a tendency for both students and researchers to associate words of a given valence with auras of the same valence, i.e., negative concepts tend to have a negative aura whereas positive concepts tend to have a positive aura. This indicates that the forma mentis networks of researchers and students are on 
average highly clustered in neighborhoods of words with similar valence attributes. Deviations from this general trend, such as negative words in the positive aura of a positive word, can be informative of the way in which a given population perceives STEM subjects.

\section{Forma Mentis networks highlight differing stances towards STEM subjects}

In this section we focus our attention on the semantic content of associations. Figure 2 reports the attribute and aura of the 10 words that were always provided to participants as cues (see Methods). Positive (negative) words are highlighted in cerulean (red) for both students (top panel) and researchers (lower panel). Researchers associated STEM-related words with mainly positive concepts, whereas students associated STEM-related words with both positive and negative concepts.

The analysis of individual words reveals that researchers perceived almost all the 10 STEM-related words as positive concepts. On the other hand, students perceived words such as "complex", "physics", "mathematics", "school" and "system" as negative concepts. Furthermore, the network structure of forma mentis networks highlighted additional critical differences in the way that students and researchers attributed positive or negative auras of valence to such negatively perceived STEM words. Specifically, students associated concepts such as "physics" and "mathematics" to other negative concepts, surrounding STEM words of quantitative disciplines with a negative valence aura. The ratio of negative to positive concepts is particularly high in the case of "mathematics", where almost $43 \%$ of associations were to other negative concepts. These patterns were absent in the forma mentis network of researchers. This comparison suggests that the presence of negative auras attributed to some STEM-related concepts is not merely a consequence of the network construction but reflects the negative stance that students have towards quantitative disciplines such as physics and mathematics.

However, it is worth noticing how the data also indicates that students did not perceive all STEM subjects as negative. In fact, concepts such as "biology" and "university" were perceived as positive and were surrounded by a positive valence aura 

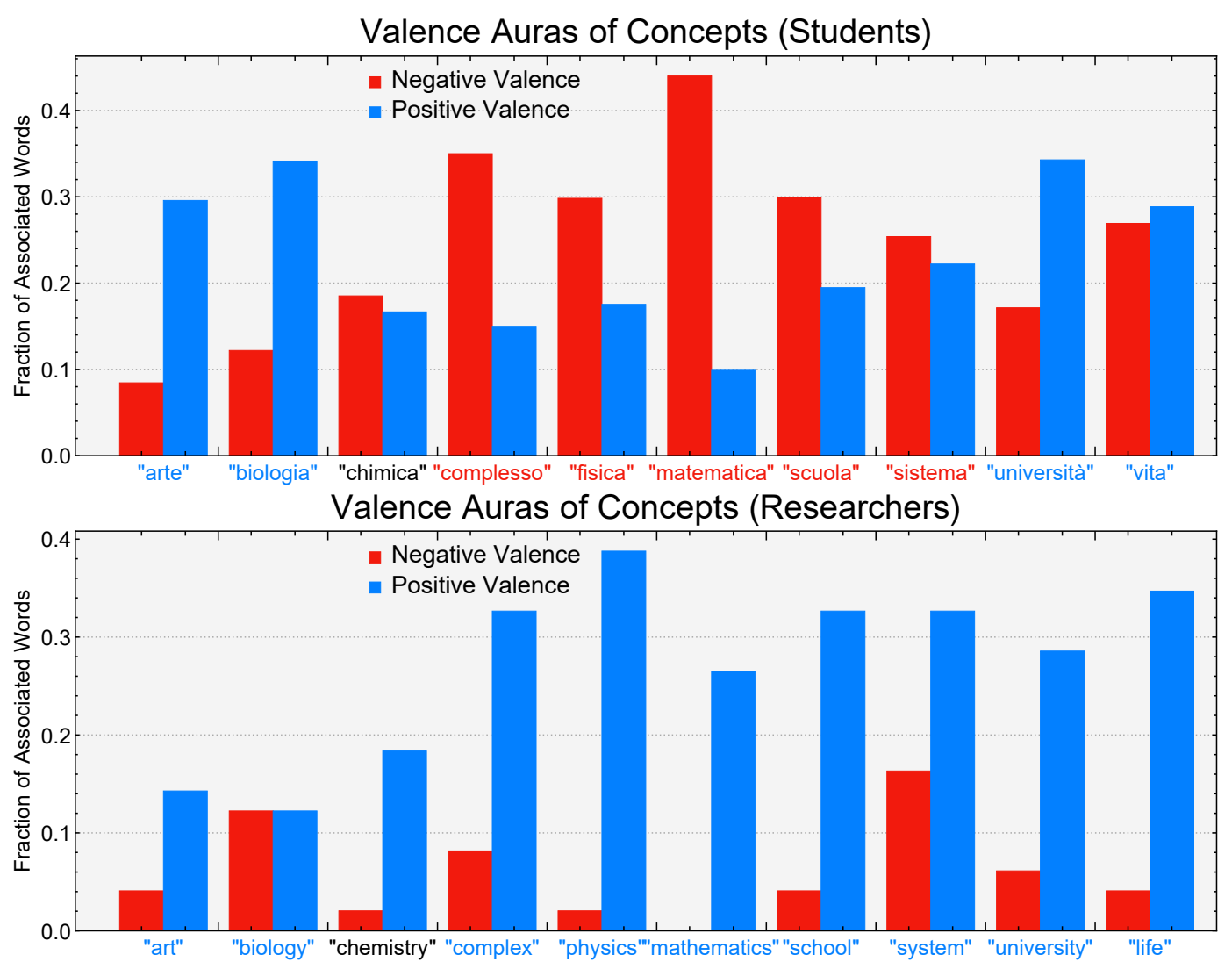

Figure 2. Valence auras identify a negative stance of students towards

specific STEM subjects. Top panel: Fraction of neighbors of concepts having a positive or a negative average valence. Concepts with positive valence are reported in blue. Concepts with negative valence are reported in red. Students perceive quantitative scientific subjects such as Mathematics and Physics negatively. Students also attributed a negative aura to these disciplines, i.e., they associated Physics mainly with other negative, rather than positive, concepts. The auras of negativity were not aimed towards all STEM subjects, since biology was perceived as positive and surrounded by an aura of positive valence. Bottom panel: Valence auras for researchers. Notice that all essential concepts were perceived as positive and surrounded by auras of positive valence.

in the students' forma mentis network. This contrast suggests that the aversion of STEM-related concepts might be related to the quantitative disciplines that underlie the scientific method used in these fields. Figure 3 shows the neighborhoods of "university" in the filtered forma mentis network of students (left) and researchers 
(right). Notice that "university" is perceived as positive and surrounded by other positive concepts such as "degree", "study", "work", and "specialisation". Even the word "researcher" is positively perceived by students, indicating a positive stance towards the general concepts of research and education. Importantly, students strongly associated the concepts of "studying" and "work", as indicated by the presence of this connection in the statistically filtered FMN. This result suggests that students might be aware of the positive impact of education has with respect to future success in the job market (Marginson et al., 2013; Rothwell, 2013).
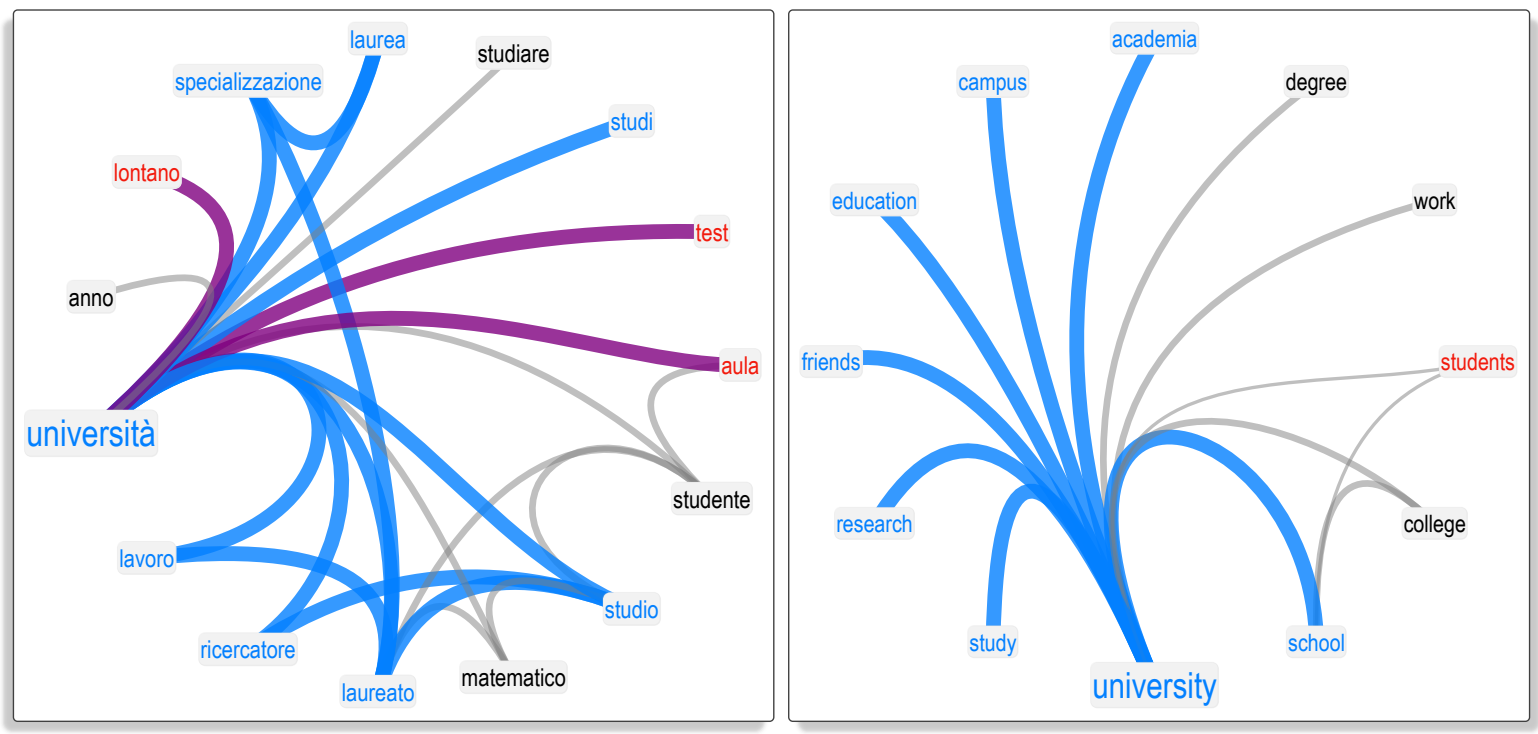

Figure 3. Neighborhoods in forma mentis networks determine the valence aura of concepts. Examples of the forma mentis networks in the neighborhood of "university" for students (left) and researchers (right). In a forma mentis network, nodes have valence attributes, i.e. "positive" (cerulean), "neutral" (grey) and "negative" (red). Links are weighted based on the number of participants providing a given association between concepts. Links between positive (negative, neutral, opposing) concepts are cerulean (red, grey, purple). The above examples include only associations provided by at least two participants. The neighbors surrounding a given word, together with their valence attributes, constitute the valence aura of that word. Both students and researchers perceive "university" as a positive concept and surround it with a positive aura. 
Figure 4 shows the neighborhoods of "physics" (top) and "mathematics" (bottom) in the filtered forma mentis network of students (left) and the FMN of researchers (right). Notice how concepts such as "physics" or "mathematics" gave rise to mostly negative associations in the students' population. The hierarchical edge bundling visualisation implemented in Mathematica 11.3 highlights that negative associations tend to cluster together, in agreement with the above clustering analysis. An inspection of the semantic content of the neighborhood for "mathematics" reveals the presence of clusters of negative concepts associated with the topic of calculus and geometry. Interestingly, most of these negative concepts were concrete tools and methodologies used in mathematics (e.g. "algorithm", "derivative", "graph", "theorem"), rather than abstract, more general terms such as "complexity". A similar result holds for "physics" (e.g., "function", "test", "integral"). A closer look at the semantic information embedded in the negative aura surrounding "physics" and "mathematics" provides preliminary evidence that the negative perception students have of these subjects may come predominantly from a negative perception of the quantitative tools usually taught in schools. In other words, the negative aura surrounding "mathematics" or "physics" does not come from a general negativity towards the whole educational system but rather from specific, concrete elements of teaching curricula. Improving the appreciation of students towards these concrete tools (e.g. "algorithm", "graph", "function") might have a beneficial effect on the perception that students have of "mathematics" or "physics", given the average trend reported above indicating that positive concepts tend to be surrounded by positive auras. However, it is important to note that our study by itself cannot either prove or disprove a causal link about concepts being perceived as positive because of their positive auras and additional research is required.

However, it is important to underline that although "mathematics" and "physics" displayed negative auras, in both the unfiltered and filtered forma mentis networks students were able to associate these subjects to "science", which is perceived as a positive concept (see also Figure 5). This link may indicate that students are aware of the importance of quantitative disciplines for the advancement of science. 

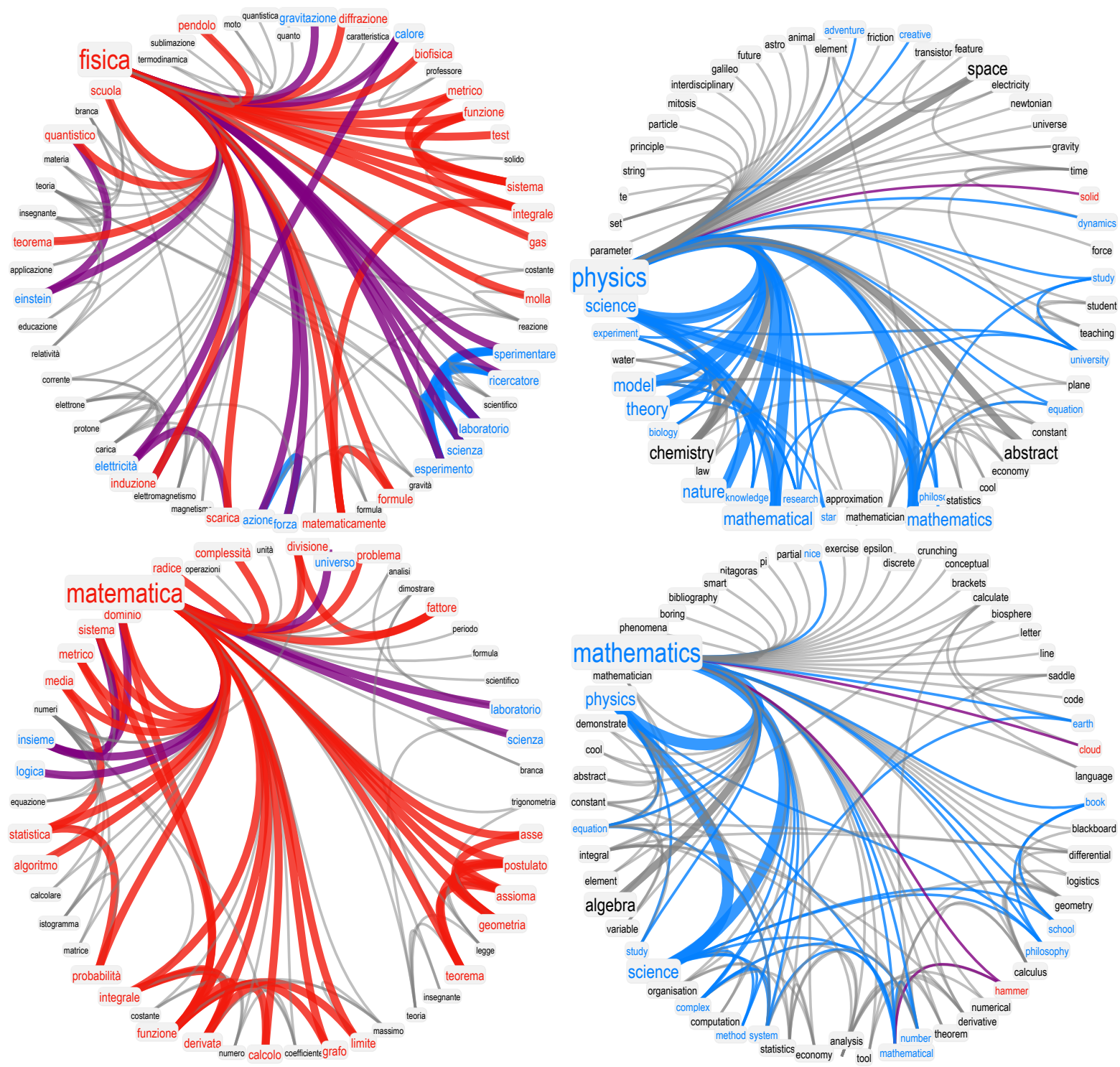

Figure 4. Mathematics and physics are perceived differently by students and

researchers.. The neighborhoods of "physics" (top) and "mathematics" (bottom) for students (left) and researchers (right). Red links indicate associations between concepts of negative valence. Stronger, more frequent associations are thicker. Students not only perceive "mathematics" and "physics" as negative concepts but also surround them with strongly negative auras of valence. This phenomenon is absent in the forma mentis network of researchers, indicating a critical negative attitude of students towards STEM quantitative subjects. Notice that for both physics and maths in students the negative aura comes mainly from clusters of specific concepts relating to specific tools (e.g. "derivative", "test", "integral"). 
Forma mentis networks further highlight the critical negative perception of students towards "mathematics" and "physics". As reported in Figure 5, those are the only negative concepts in the otherwise positive valence aura surrounding "science". This contrast indicates that, although on a technical level students were aware about the links between quantitative disciplines and science, they were unable to transfer their positive perception of science to the building blocks of the scientific method.
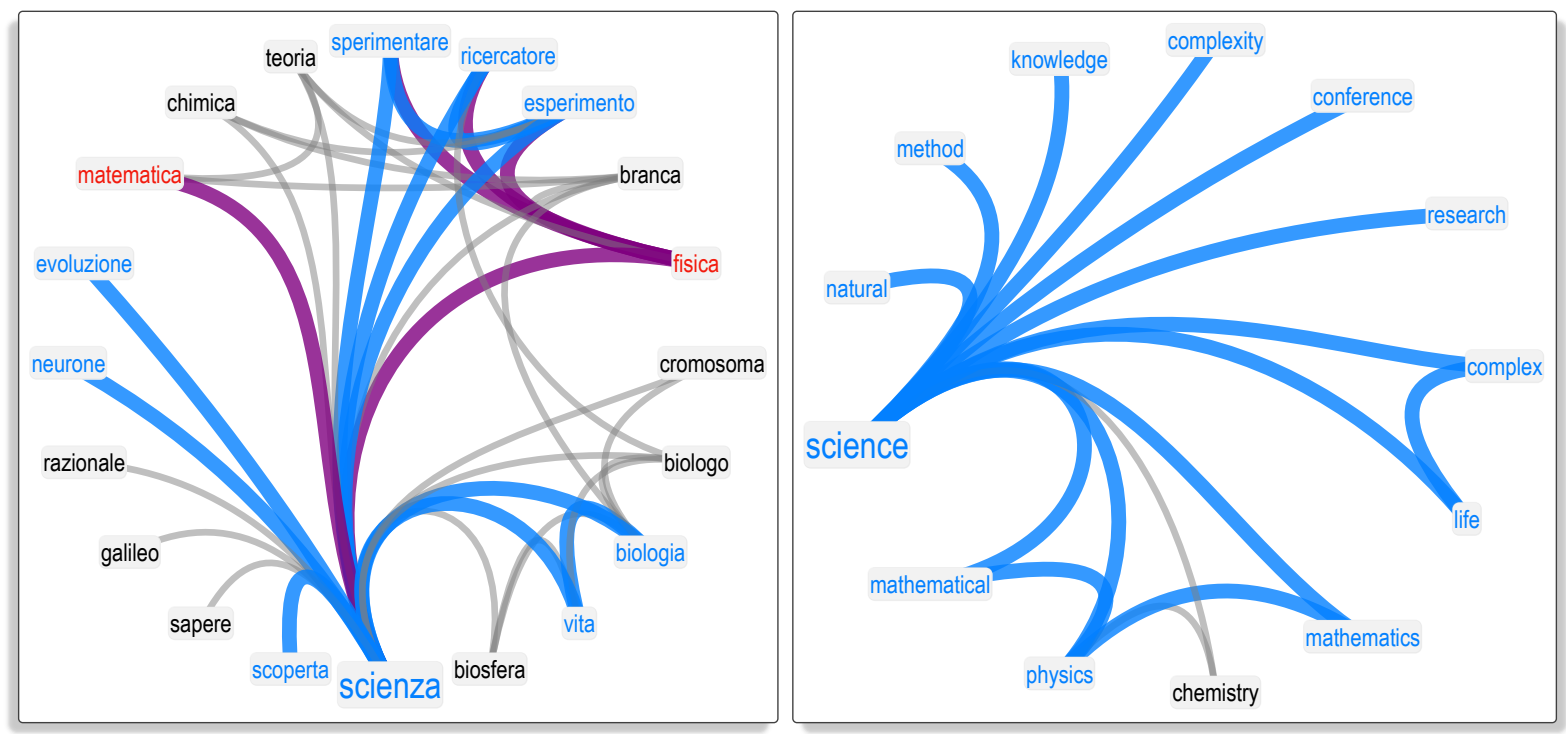

Figure 5. Maths and physics are the main negative outliers in the otherwise positive aura of "science" as perceived by students. "Science" was never provided as a cue either to students or to researchers. It was one of the associations provided by participants. Neighborhoods of "science" in the forma mentis networks of students (left) and researchers (right): although students perceived science and other STEM subjects as positive concepts, surrounding science itself with an aura of positive valence, students also perceived mathematics and physics as negative concepts. This plot included only statistically significant free associations.

\section{Discussion}

Forma mentis networks represent an innovative combination of free associations, i.e. words that are elicited in response to cue words (Nelson et al., 2004), with additional affective information of each word's valence (Posner et al., 2005; Warriner et al., 2013), i.e. how positively or negatively a given concept is perceived. 
From a methodological perspective, this combination of two sources of linguistic information fills a gap in the literature of language networks modelling the mental lexicon (Siew et al., 2018), where conceptual units are considered only in terms of their semantic features (De Deyne et al., 2013; De Deyne et al., 2018; Kenett et al., 2014; Kenett et al., 2018; Steyvers \& Tenenbaum, 2005), and not their valence. Given that recent evidence indicates that emotions deeply influence language processing and memory even at nonconscious levels (Adelman \& Estes, 2013; Gaillard et al., 2006), forma mentis networks represent a natural extension of semantic representations of the mental lexicon that includes affective attributes of individual concepts.

The combination of sentiment and semantic structure leads to the definition of valence auras, in which concepts are not isolated affective entities but rather interacting emotional elements of an associative network. The empirical evidence reported in this work shows a tendency for concepts of a given valence to cluster with words of the same valence. This assortative mixing of links is known as homophily in social network analysis (McPherson, Smith-Lovin, \& Cook, 2001) and represents a tendency for units to link mainly to other units sharing similar features. To the best of our knowledge, our work represents the first evidence of emotional homophily in the human mental lexicon. This emotional homophily leads to concepts of a given valence being surrounded by other concepts with the same valence. By cross-validating our data with independent datasets of affective norms (Fairfield et al., 2017; Warriner et al., 2013), we showed that negative words surrounded by a negative aura elicited a higher arousal compared to negative words surrounded by any (neutral, negative, or positive) aura. This difference could be interpreted in terms of individual concepts exerting an influence over their associated neighbors, with negative words increasing levels of emotional intensity elicited during cognitive processing such that negative words surrounded by other negative words have higher arousal ratings than expected. This interaction between emotional processing and the structure of semantic memory itself should be further explored in future psycholinguistic research.

In the present paper, emotional homophily in forma mentis networks provides us 
with new ways of detecting the stance of a given population. Our application of the forma mentis networks has led to three main insights into the way that students and researchers perceive STEM topics.

First, we used the network structure of FMNs to identify and define the "aura" of a concept, i.e., its first neighbors in the association network created by the participants. An analysis of valence auras, in addition to the words' individual valence attributes, uncovers a clear pattern in the students' FMN in which negative words surrounded by a negative aura were correlated with higher arousal ratings. Moreover, words of the same valence tended to cluster together, indicating a conceptual organization that may have been shaped by the valences of words. Overall, it appeared that the students' stance towards STEM subjects is mixed, combining both positive and negative stances, whereas the researchers' stance toward STEM was predominantly positive.

Second, at the semantic level, the comparison between students' and researchers' networks led both to unexpected similarities as well as interesting contrasts. On the one hand, Italian high school students are almost as skilled as experts in relating key concepts of STEM subjects to "science", such that "science" itself was a key concept in their forma mentis network. This finding provides evidence that at a global level Italian students possess a good technical awareness of STEM subjects in comparison with STEM professionals. Analogously, a comparable level of student competence has also been reported in other educational systems, such as the Finnish one, by independent studies from Koponen and Maija using concept maps (Ismo T. Koponen \& Nousiainen, 2019).

Furthermore, students perceived concepts such as "mathematics" and "physics" not only as negative, but surrounded by negative auras as well, whereas words such as "science" were positively perceived by both students and researchers. This dichotomy between the positive aura of science and the negative auras of mathematics and physics is absent in the group of STEM professionals, and it suggests that students might not be sufficiently aware of the connections between science, its methods and its applications. Another interpretation of the negative auras surrounding mathematics, physics and 
other concepts like statistics (cf. Supplementary Figure 3) might relate to emotional homophily and anxiety. As discussed above, negative concepts surrounded by a negative aura tended to also have higher arousal and lower valence ratings (based on external datasets). In the circumplex model of emotions (Posner et al., 2005), higher arousal and negative valence correspond to emotions of stress and anxiety. Hence, the negative emotional auras of physics, maths and statistics in the forma mentis of students suggest that high school students may experience stress and anxiety toward such disciplines. This finding is supported by an increasingly developing literature about mathematics anxiety (Ashcraft, 2002), physics anxiety (Laukenmann et al., 2003) and even statistics anxiety (Siew et al., 2019) affecting students' learning at the high school level and continuing even through university. In this way, forma mentis networks might represent an innovative way of quantifying science anxiety in student populations, potentially working in synergy with other network studies quantifying anxiety levels with complex networks (Siew et al., 2019) and psychological methodologies (Ashcraft, 2002).

In spite of the bleak perception of mathematics and physics, the students showed a positive perception of both "university" and "researcher", which indicates an awareness of the importance of education for their professional future.

Third, valence auras allowed us to hypothesize viable reasons for the disaffection towards mathematics and physics beyond anxiety: Words with a negative valence in those subjects' aura are mostly mathematical tools and techniques, such as "integral" or "function". This result highlights how the negative stance on physics and mathematics might not originate from a wider distrust of the subjects themselves but perhaps from the difficulty in seeing the value of these techniques, particularly when devoid of interdisciplinary connections. This result opens possible avenues for intervention in education by, for instance, helping students to embed mathematics and physics within a richer network of conceptual associations.

What might be missing from the educational curriculum of the students participating in this study is an emphasis on the connections between quantitative disciplines and real-world settings. Beyond sterile arguments that a discipline should be 
appreciated because of its inner beauty, our results suggest that even at the high school level, educators should provide as many opportunities as possible for students to discover the beauty of STEM subjects and learn about the implications of mathematical modelling of real-world systems, as previously highlighted in the relevant educational literature (C. B. Cramer et al., 2018; Resnick, 1997; Sayama et al., 2016; van der Cingel, 2018).

A positive stance towards mathematics and physics among early career complexity researchers could be due to the fact that many real-world models of complexity science are grounded in quantitative disciplines such as mathematics and physics (Mitchell, 2009; Resnick, 1997). However, a large proportion of complex systems scientists do not identify as mathematicians or physicists, and come from a diverse range of disciplines, including biology, economics, chemistry, archaeology, art, psychology, and the social sciences. The application of quantitative tools to aid the understanding of complex systems might have led to a positive perception of these concepts among professionals, as reflected in their forma mentis network.

Complexity science seems to be a natural candidate for improving the perception of STEM subjects among students. Indeed, previous attempts at building network science courses at the high school level are generally met with interest by high school students (cf. C. B. Cramer et al., 2018; C. Cramer et al., 2018; van der Cingel, 2018). In addition, the Complex Forma Mentis project (www.complexmentis.com Last Accessed: 19 February 2019) provided seminars about complexity science at the high school level that were met with strong interest. Although these initiatives are still early in their implementation, the framework of complexity science may prove to be useful in helping students learn about how the technical aspects of STEM disciplines can be used to address important societal problems, and as a result improve their perception of technical, sometimes obscure, concepts related to mathematical theory and physics.

Another reason behind the dissonant stance of students towards physics and mathematics might be related to a lack of creativity. Recently, Valenti and colleagues (Valenti et al., 2016) measured the implicit attitudes towards science in a population of 
students and found that the increase of scientific rigour is accompanied by a decrease in associating science with creativity. In the FMN of researchers, "art" is connected to "creative" and "science", whereas these concepts were disconnected in the forma mentis network of students. Researchers also associated "physics" with "creative", an association that is missing in students' FMN. These missing links further underline the importance for students to build a more complete and broader perception of STEM subjects, focusing on the creative process behind science and its real-world, complex implications.

Nevertheless, the current approach of FMNs has some limitations that we discuss below. The most prominent one is that forma mentis networks operate at the population level, as is common in psycholinguistic approaches relying on free association networks (De Deyne et al., 2013; Nelson et al., 2004) or in educational studies using concept maps (Ismo T. Koponen \& Nousiainen, 2018; Ismo T Koponen \& Pehkonen, 2010; Sayama et al., 2016). Hence, the above patterns have to be interpreted in terms of average trends, as individual students might differ from the aggregated pattern. However, recent approaches have constructed association networks at the level of individuals (Kenett et al., 2018; Siew, 2018) and even reported how individualized free association networks were predictive of creativity levels (Kenett et al., 2018) or knowledge mastery (Siew, 2018). With larger sample sizes and a more substantive free association task (leading to denser networks), building forma mentis networks for the individuals represents an exciting research direction for the future.

Another limitation is the experimental effort in engaging participants within a cognitive task, compared to the relative ease of mining online data from social media in order to infer stance. A possible solution could be the use of social media mining to extract semantic associations for forma mentis networks, analogous to the semantic networks of concept co-occurrences in Twitter by Stella, Ferrara, and De Domenico, 2018. Although this might decrease the difficulty of building a network representation of the mental lexicon of a given population, co-occurrences of words in text are different from free associations and provide different cognitive information with regards to language acquisition and use. For instance, in Stella et al. (Stella et al., 2017), free 
associations proved to be more predictive of early word learning compared to word co-occurrences in child directed speech. Hence, despite the difficulty of collection free associations, we argue that free associations provide important insights into the structure of the mental lexicon and that such data are worth the time and cost of data collection.

\section{Conclusions}

This article introduced the new methodology of forma mentis networks and demonstrated its potential to identify contrasting stances in different populations. A forma mentis network consists of words as nodes, each with a valence attribute, and free associations as links. Rather than being based on automatic natural language processing, these networks directly access the mental lexicon of human participants, addressing the orthogonal influences of semantic knowledge and emotional affect that drive the processing of information (Siew et al., 2018; Vitevitch, 2008; Vitevitch et al., 2018) and its consequent reactions (Adelman \& Estes, 2013; Christensen et al., 2018; Gaillard et al., 2006; Zurn \& Bassett, 2018).

We found substantial differences in the stances of young researchers in complexity science and high school students towards STEM concepts such as physics and mathematics. Students tended to surround these concepts with a negative emotional aura, which could related to a perceived anxiety toward these subjects (cf. Ashcraft, 2002; Posner et al., 2005; Siew et al., 2019). This negative emotional aura was absent in the forma mentis of researchers. Furthermore, the words with a negative valence in the students' neighborhoods were mostly that of mathematical tools, such as "integral" or "function". This result highlighted how the students' negative stance toward physics and mathematics might predominantly originate from an arid view of the tools and methods used in mathematics and physics, which students (but not researchers) perceived as deprived of more interdisciplinary and creative connections.

This quantitative evidence opens new avenues for intervention in education: Encouraging students to incorporate mathematics and physics into a richer association 
network and drawing new connections to other concepts in the scientific realm represent promising pathways to change their stance. In that lies the potential of the forma mentis network approach-by providing a map of the students' mental lexicon, it is able to show which and where new meaningful links, i.e. associations, could be constructed to maximize the effectiveness of future intervention policies and outreach programmes.

\section{Acknowledgements}

The authors acknowledge the Winter Workshop on Complex Systems, the NetSciEd community, the Mediterranean School on Complex Networks and the schools that enthusiastically joined the project: IISS "E. Medi”, Galatone (principal: Davide Cammarota), Liceo “G. Banzi Bazoli”, Lecce (principal: Antonella Manca), IISS "E. Fermi", Lecce (principal: Giuseppe Russo). Many thanks also to Antonella Conte, Mason A. Porter and Hiroki Sayama for their kind feedback on the manuscript.

\section{Appendix}

This Appendix outlines the role played by idiosyncratic associations in forma mentis networks, i.e. associations there were provided by less than two different participants in the free association task. When using free associations for modelling the structure of the mental lexicon, idiosyncratic associations are usually filtered out and the focus is given to more frequent associations between concepts (Kenett et al., 2014). However, also idiosyncratic associations can provide additional information about how individuals perceive the external world (Nelson et al., 2004), given that associations' idiosyncracy might be just an outcome of the limited number of interviewed participants in a given free association task. Hence, also idiosyncratic associations can be relevant for the above investigation of stance from FMNs.

Supplementary Figure S1 reports forma mentis networks of students and researchers in the neighborhood of "university" including also idiosyncratic associations. In the students' forma mentis network, important concepts like "courses", "curricula" or "academia" are linked to "university" only when idiosyncratic associations are considered. 
Supplementary Figure S2 reports the neighborhood of "science" in the students' FMN including also idiosyncratic associations. Even when the noise introduced by idiosyncratic associations is considered, science is mostly surrounded by a positive emotional aura, where negative concepts are mainly those related or clustering around physics and maths. Hence, idiosyncratic associations do not alter the emotional gap discussed in the main text about STEM stance.

Supplementary Figure S3 reports the neighborhood of "statistics" in the students' and researchers' FMNs including also idiosyncratic associations. Beyond mathematics and physics, also other STEM concepts like statistics are surrounded by a negative aura in the students' FMN, a negative perception that is absent in the researchers' forma mentis network. 


\section{References}

Adelman, J. S. \& Estes, Z. (2013). Emotion and memory: a recognition advantage for positive and negative words independent of arousal. Cognition, 129(3), 530-535. Aitchison, J. (2012). Words in the mind: an introduction to the mental lexicon. John Wiley \& Sons.

Amancio, D. R. (2015). A complex network approach to stylometry. PloS one, 10(8), e0136076.

Ashcraft, M. H. (2002). Math anxiety: personal, educational, and cognitive consequences. Current directions in psychological science, 11(5), 181-185.

Biber, D. \& Finegan, E. (1989). Styles of stance in english: lexical and grammatical marking of evidentiality and affect. Text-interdisciplinary journal for the study of discourse, 9(1), 93-124.

Christensen, A. P., Kenett, Y. N., Cotter, K. N., Beaty, R. E., \& Silvia, P. J. (2018). Remotely close associations: openness to experience and semantic memory structure. European Journal of Personality, 32(4), 480-492.

Cramer, C. B., Porter, M. A., Sayama, H., Sheetz, L., \& Uzzo, S. M. (2018). Network science in education: transformational approaches in teaching and learning (1st). Springer Publishing Company, Incorporated.

Cramer, C., Gera, R., Panagakou, E., Porter, M. A., Sayama, H., Sheetz, L., ... Uzzo, S. (2018). Proceedings of NetSciEd 2018. OSF Preprints.

De Deyne, S., Navarro, D. J., \& Storms, G. (2013). Better explanations of lexical and semantic cognition using networks derived from continued rather than single-word associations. Behavior research methods, 45(2), 480-498.

De Deyne, S., Navarro, D. J., Perfors, A., Brysbaert, M., \& Storms, G. (2018). The "small world of words" english word association norms for over 12,000 cue words. Behavior research methods, 1-20.

Fairfield, B., Ambrosini, E., Mammarella, N., \& Montefinese, M. (2017). Affective norms for italian words in older adults: age differences in ratings of valence, arousal and dominance. PloS one, 12(1), e0169472. 
Gaillard, R., Del Cul, A., Naccache, L., Vinckier, F., Cohen, L., \& Dehaene, S. (2006). Nonconscious semantic processing of emotional words modulates conscious access. Proceedings of the National Academy of Sciences, 103(19), 7524-7529.

Gray, B. \& Biber, D. (2012). Current conceptions of stance. In Stance and voice in written academic genres (pp. 15-33). Springer.

Hills, T. T. \& Siew, C. S. Q. (2018). Filling gaps in early word learning. Nature Human Behaviour, 2(9), 622.

Kenett, Y. N., Anaki, D., \& Faust, M. (2014). Investigating the structure of semantic networks in low and high creative persons. Frontiers in human neuroscience, 8 , 407.

Kenett, Y. N., Levi, E., Anaki, D., \& Faust, M. (2017). The semantic distance task: quantifying semantic distance with semantic network path length. Journal of Experimental Psychology: Learning, Memory, and Cognition, 43(9), 1470.

Kenett, Y. N., Levy, O., Kenett, D. Y., Stanley, H. E., Faust, M., \& Havlin, S. (2018). Flexibility of thought in high creative individuals represented by percolation analysis. Proceedings of the National Academy of Sciences, 115(5), 867-872.

Koponen, I. T. [Ismo T.] \& Nousiainen, M. (2018). Concept networks of students' knowledge of relationships between physics concepts: finding key concepts and their epistemic support. Applied Network Science, 3(1), 14.

Koponen, I. T. [Ismo T.] \& Nousiainen, M. (2019). Pre-service teachers' knowledge of relational structure of physics concepts: finding key concepts of electricity and magnetism. Education Sciences.

Koponen, I. T. [Ismo T] \& Pehkonen, M. (2010). Coherent knowledge structures of physics represented as concept networks in teacher education. Science $\mathbb{E}$ Education, 19(3), 259-282.

Krapp, A. \& Prenzel, M. (2011). Research on interest in science: theories, methods, and findings. International journal of science education, 33(1), 27-50.

Laukenmann, M., Bleicher, M., Fuß, S., Gläser-Zikuda, M., Mayring, P., \& von Rhöneck, C. (2003). An investigation of the influence of emotional factors on 
learning in physics instruction. International Journal of Science Education, 25(4), $489-507$.

Marginson, S., Tytler, R., Freeman, B., \& Roberts, K. (2013). Stem: country comparisons: international comparisons of science, technology, engineering and mathematics (stem) education. final report.

McPherson, M., Smith-Lovin, L., \& Cook, J. M. (2001). Birds of a feather: homophily in social networks. Annual review of sociology, 27(1), 415-444.

Mitchell, M. (2009). Complexity: a guided tour. Oxford University Press.

Mohammad, S. M., Sobhani, P., \& Kiritchenko, S. (2017). Stance and sentiment in tweets. ACM Transactions on Internet Technology (TOIT), 17(3), 26.

Mohammad, S., Kiritchenko, S., Sobhani, P., Zhu, X., \& Cherry, C. (2016). Semeval-2016 task 6: detecting stance in tweets. In Proceedings of the 10th international workshop on semantic evaluation (semeval-2016) (pp. 31-41).

Nelson, D. L., McEvoy, C. L., \& Schreiber, T. A. (2004). The university of south florida free association, rhyme, and word fragment norms. Behavior Research Methods, Instruments, \& Computers, 36(3), 402-407.

Osborne, J., Simon, S., \& Collins, S. (2003). Attitudes towards science: a review of the literature and its implications. International journal of science education, 25(9), 1049-1079.

Posner, J., Russell, J. A., \& Peterson, B. S. (2005). The circumplex model of affect: an integrative approach to affective neuroscience, cognitive development, and psychopathology. Development and psychopathology, 17(3), 715-734.

Resnick, M. (1997). Turtles, termites, and traffic jams: explorations in massively parallel microworlds. Mit Press.

Rothwell, J. (2013). The hidden stem economy. Brookings.

Sayama, H., Cramer, C., Porter, M. A., Sheetz, L., \& Uzzo, S. (2016). What are essential concepts about networks? Journal of Complex Networks, 4(3), 457-474.

Siegel, D. J. (2015). The developing mind: how relationships and the brain interact to shape who we are. Guilford Publications. 
Siew, C. S. Q. (2018). Using network science to analyze concept maps of psychology undergraduates. Applied Cognitive Psychology.

Siew, C. S. Q., McCartney, M. J., \& Vitevitch, M. S. (2019). Using network science to understand statistics anxiety among college students. Scholarship of Teaching and Learning in Psychology.

Siew, C. S. Q., Wulff, D. U., Beckage, N., \& Kenett, Y. (2018). Cognitive network science: a review of research on cognition through the lens of network representations, processes, and dynamics.

Somasundaran, S. \& Wiebe, J. (2010). Recognizing stances in ideological on-line debates. In Proceedings of the naacl hlt 2010 workshop on computational approaches to analysis and generation of emotion in text (pp. 116-124). Association for Computational Linguistics.

Stella, M. (2019). Modelling early word acquisition through multiplex lexical networks and machine learning. Big Data and Cognitive Computing, 3(1), 10.

Stella, M., Beckage, N. M., \& Brede, M. (2017). Multiplex lexical networks reveal patterns in early word acquisition in children. Scientific reports, 7, 46730.

Stella, M., Beckage, N. M., Brede, M., \& De Domenico, M. (2018). Multiplex model of mental lexicon reveals explosive learning in humans. Scientific reports, 8(1), 2259.

Stella, M., Ferrara, E., \& De Domenico, M. (2018). Bots increase exposure to negative and inflammatory content in online social systems. Proceedings of the National Academy of Sciences, 115(49), 12435-12440.

Steyvers, M. \& Tenenbaum, J. B. (2005). The large-scale structure of semantic networks: statistical analyses and a model of semantic growth. Cognitive science, $29(1), 41-78$.

Valenti, S., Masnick, A., Cox, B., \& Osman, C. (2016). Adolescents' and emerging adults' implicit attitudes about stem careers:" science is not creative". Science Education International, 27(1), 40-58. 
Valenzuela Castellanos, M. F., Pérez Villalobos, M., Bustos, C., \& Salcedo Lagos, P. (2018). Cambios en el concepto aprendizaje de estudiantes de pedagogía: análisis de disponibilidad léxica y grafos. Estudios filológicos, (61), 143-173.

van der Cingel, P. (2018). How to educate navigators in a complex world: making a case in higher professional education in the netherlands. Complexity, governance and networks, 4(1).

Vitevitch, M. S. (2008). What can graph theory tell us about word learning and lexical retrieval? Journal of Speech, Language, and Hearing Research.

Vitevitch, M. S., Siew, C. S. Q., \& Castro, N. (2018). Spoken word recognition. The Oxford Handbook of Psycholinguistics, 31.

Warriner, A. B., Kuperman, V., \& Brysbaert, M. (2013). Norms of valence, arousal, and dominance for 13,915 english lemmas. Behavior research methods, 45(4), $1191-1207$.

Zurn, P. \& Bassett, D. S. (2018). On curiosity: a fundamental aspect of personality, a practice of network growth. Personality Neuroscience, 1. 

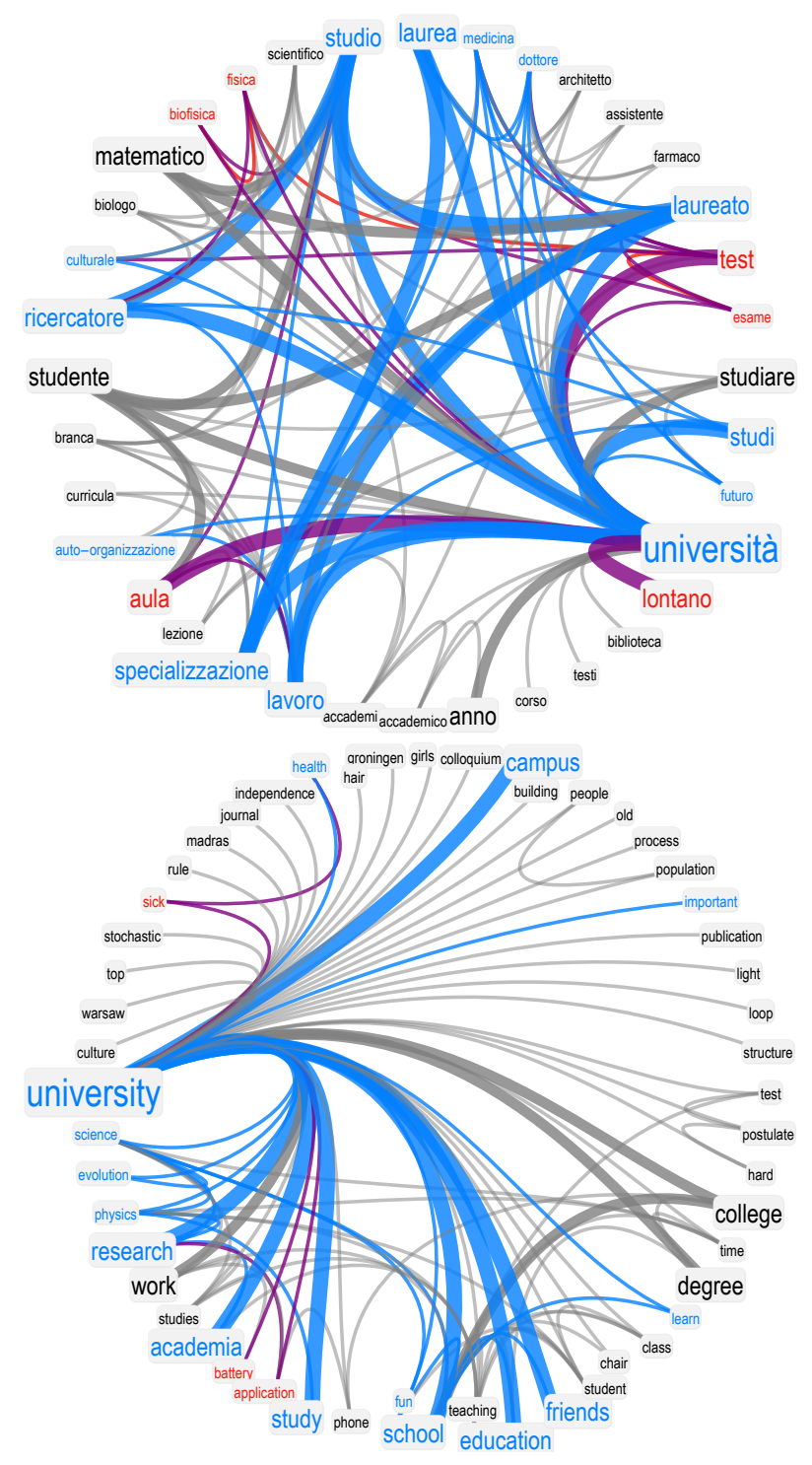

Figure 6. Supplementary Figure: Idiosyncratic interactions provide

additional network structure. Examples of the unfiltered forma mentis networks in the neighborhood of "university" for students (left) and researchers (right), including also idiosyncratic associations made only by one participant. Associations provided by two or more participants are marked thicker, with the same colour scheme of Figure 3 in the main text. Notice that some associations might be idiosyncratic only because of the smaller pool of participants and they might still provide meaningful additional semantic content. 


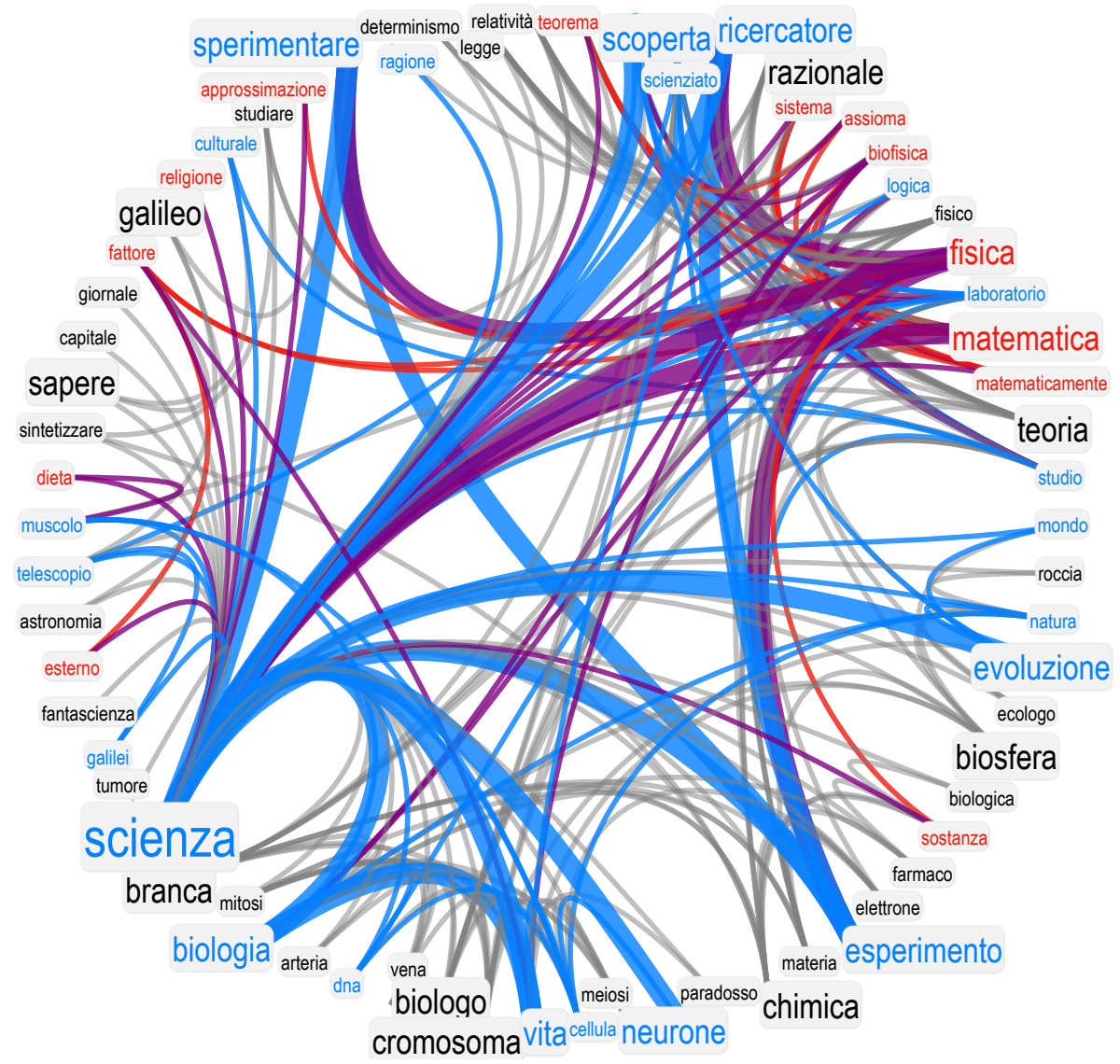

Figure 7. Supplementary Figure: Including idiosyncratic associations highlights additional negative concepts semantically related to maths, physics and science. neighborhood of "science" as perceived by students including also idiosyncratic associations. Even in this case, most of the negative concepts associated to science are semantically related to maths and physics. The fact that the associations science-maths and science-physics indicates that students have the necessary knowledge for relating scientific discovery to quantitative STEM subjects. However the valence analysis clearly indicates a gap in diffusing the aura of positivity of science also to maths and physics. 

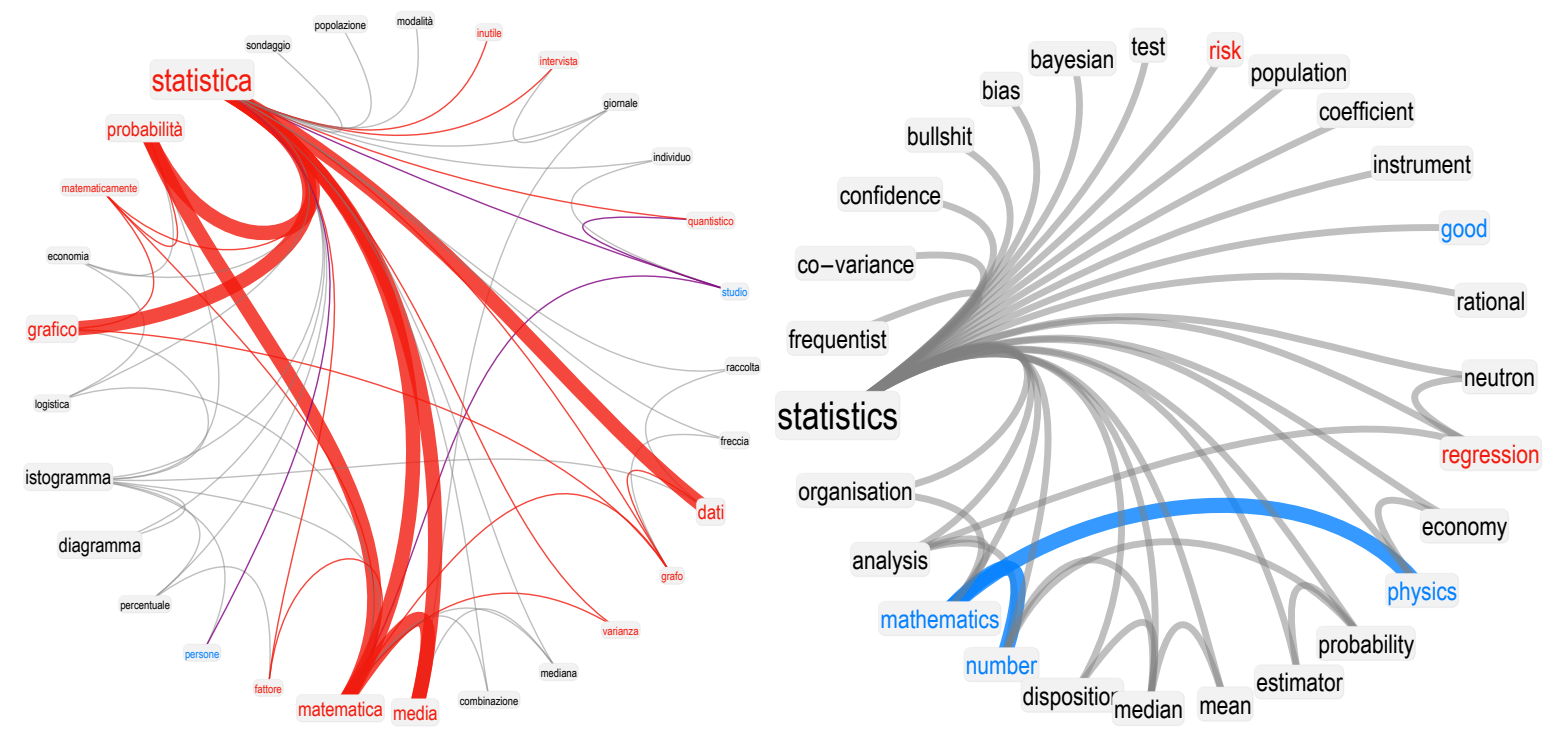

Figure 8. Supplementary Figure: Also statistics is surrounded by a negative aura in students' FMN. Neighborhood of "statistics" as perceived by students (left) and researchers (right) including also idiosyncratic associations (thin links) and non-idiosyncratic associations (thicker links). Statistics is surrounded by a negative aura in the students' filtered FMN network, whereas researchers perceive it mainly as a neutral concept. 